CORRECTION

https://doi.org/10.1038/s41586-018-0827-2

\title{
Author Correction: A population of luminous accreting black holes with hidden mergers
}

Michael J. Koss, Laura Blecha, Phillip Bernhard, Chao-Ling Hung, Jessica R. Lu, Benny Trakhtenbrot, Ezequiel Treister, Anna Weigel, Lia F. Sartori, Richard Mushotzky, Kevin

Schawinski, Claudio Ricci, Sylvain Veilleux \& David B. Sanders

Correction to: Nature https://doi.org/10.1038/s41586-018-0652-7, published online 07 November 2018.

In this Letter, the surname of author Benny Trakhtenbrot was misspelled 'Trakthenbrot'. The affiliation for author Sylvain Veilleux should have been 'Department of Astronomy and Joint Space-Science Institute, University of Maryland, College Park, MD, USA' instead of 'Instituto de Astrofísica...Chile. In addition, we omitted to cite a reference ${ }^{1}$ by a co-author in the Abstract sentence 'Recent observations have shown that a black hole is likely to become heavily obscured behind merger-driven gas and dust,..... This has been added to the reference list as ref. 9 of the original Letter, with subsequent references renumbered accordingly. Finally, the following sentence has been added to the Acknowledgements section 'We appreciate the help of R. M. McGurk with the initial night of Keck telescope observations and initial data reductions.' This Letter has been corrected online.

1. Ricci, C. et al. Growing supermassive black holes in the late stages of galaxy mergers are heavily obscured. Mon. Not. R. Astron. Soc. 468, 21 (2017). 